\title{
Eine Alternative zur COPD-Diagnostik mit Spirometrie bei Betagten?
}

Hintergrund und Fragestellung: Die Spirometrie kann beim geriatrischen COPD-Patienten Probleme machen, sowohl was das Untersuchungsverständnis als auch die korrekte Durchführung betrifft. Daher ist das Risiko für falsche Befunde relativ hoch. In ihrer cross-sektionalen Studie beschreiben die Autoren die Problematik der Spirometrie bei geriatrischen COPD-Patienten. Hierbei bewerten sie den (Zusatz-)Nutzen der forcierten oszillatorischen Technik (FOT) in Ergänzung zur herkömmlichen Methode $\mathrm{FEV}_{1}$-Bestimmung. Studienziel war es herauszuOriginalie

Tse HN, Tseng CZ, Wong KY et al. Accuracy of forced oscillation technique to assess lung function in geriatric COPD population. Int J Chron Obstruct Pulmon Dis. 2016 26;11:1105-18. finden, ob die FOT zusätzliche Informationen liefert.

Patienten u. Methoden: Patienten im mittleren Alter von 72 Jahren, deren Anamnese mit einer COPD vereinbar ist und die $\mathrm{FEV}_{1}$ bei <0,7 liegt, wurden in die Studie eingeschlossen. Zudem erfolgte eine Untersuchung mittels FOT und Plethysmografie. Die FOT erfolgte mit dem i2M der Firma Chess Medical Ghent (Belgien) und arbeitete im Bereich von $4-48 \mathrm{~Hz}$.

Ergebnisse: 158 Patienten wurden in die Studie aufgenommen. Die FOT korrelierte gut mit den übrigen Spirometriedaten, die Resistance-Daten besser als die Compliance-Daten. Es fand sich eine hohe Sensitivität und Spezifität für COPD. Auch im Vergleich mit den GOLD-Schweregraden war der Zusammenhang zwischen den herkömmlichen Methoden und der FOT gut.

Schlussfolgerung: Geriatrischen Patienten haben oft Schwierigkeiten mit herkömmlichen Lungenfunktionstests. Die FOT ist ein einfaches und akkurates Untersuchungstool, um den Schweregrad von „Luftstrombehinderungen“ in den kleinen und mittleren Bronchien zu erfassen und eine COPD bei zu beurteilen.

\section{- Kommentar von Professor Dr. med. Hans Jürgen Heppner}

\section{Manko: Studie ethnisch und geschlechtsbezogen unausgewogen}

Die guten Ergebnisse des Vergleichs von FOT und spirometrischen Parametern wurde bereits beschrieben [1], aber bisher nicht bei geriatrischen Patienten. Dies ist wichtig, da diese Patientengruppe oft funktional und kognitiv eingeschränkt und nicht in der Lage ist, den herkömmlichen forcierten Spirometrietest durchzuführen. Es ist bisher auch nicht ganz klar, wie aus dem Geriatrischen Assessment Rückschlüsse auf die Lungenfunktion bei COPD-Patienten geschlossen werden kann [2].

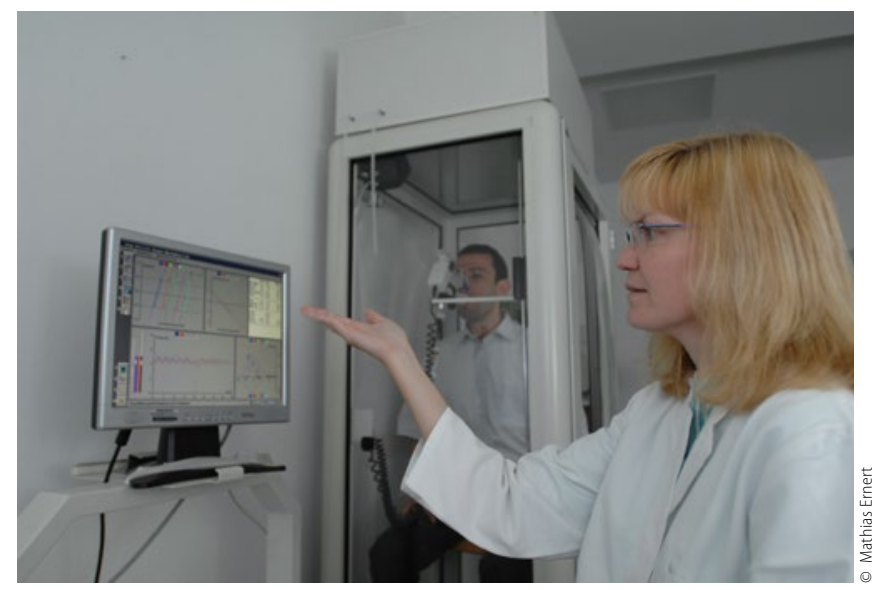

Bei der Spirometrie muss auf Anweisungen punktgenau reagiert werden, das ist für viele geriatrische Patienten ein Problem.
So bietet sich die FOT, wie sie auch in der Anästhesie perioperativ teilweise verwendet wird, als Funktionsuntersuchung bei besonderen Bedingungen an [3]. Zwar muss berücksichtigt werden, dass die Studie überwiegend mit männlichen Chinesen gemacht wurde und daher die Aussagekraft ein wenig eingeschränkt ist, jedoch konnten die Autoren zeigen, dass die FOT bei geriatrischen Patienten eine Ergänzung/Alternative zur konventionellen Spirometrie ist. Daher ist die FOT eine vielversprechende Methode bei geriatrischen COPD-Patienten, wenngleich weitere Studien dazu noch wünschenswert sind.

\footnotetext{
Literatur

1. Williamson PA, Clearie K, Menzies D et al. Lung. 2011;189(2):121-129

2. Fronhofen H, Hagen O. Z Gerontol Geriatr. 2011 Aug;44(4):245-9

3. Nakano S, Nakahira J, Sawai T et al. BMC Anesthesiol. 2016 Jul 7;16(1):32
}

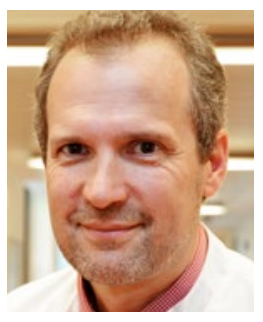

Univ.- Prof. Dr. med. Hans Jürgen Heppner

Chefarzt Geriatrische Klinik und Tagesklinik Lehrstuhl für Geriatrie Universität Witten/Herdecke Dr.-Moeller-Straße 15, 58332 Schwelm hans.heppner@uni-wh.de 